Proc. XII Int. School on Theoretical Physics — Symmetry and Structural Properties of Condensed Matter

\title{
Effect of Rashba Spin-Orbit Coupling on the Spin Polarization of Holes in Two-Dimensional GaMnAs Magnetic Semiconductors
}

\author{
S. StagraczyŃSKi ${ }^{a}$, V.K. DUGAeV ${ }^{b}$ AND J. BeRAKDAR ${ }^{a}$ \\ ${ }^{a}$ Institut für Physik, Martin-Luther-Universität Halle-Wittenberg, \\ Karl-Freiherr-von-Fritsch-Str. 3, 06120 Halle (Saale), Germany \\ ${ }^{b}$ Department of Physics and Medical Engineering, Rzeszów University of Technology, \\ al. Powstańców Warszawy 6, 35-959 Rzeszów, Poland
}

\begin{abstract}
We consider the effect of the Rashba spin-orbital coupling in two-dimensional GaAs semiconductor heavily doped with $\mathrm{Mn}$, on the spin polarization of holes. Due to the strong internal spin-orbit interaction in GaAs, the spin of a hole is not a good quantum number but the hole in some energy state has a certain mean value of spin, which can be strongly affected by the Rashba spin-orbital interaction related to the substrate for $2 \mathrm{D}$ material.
\end{abstract}

DOI: 10.12693/APhysPolA.132.189

PACS/topics: Magnetization, Rashba spin-orbit coupling

\section{Introduction}

Since the seminal theoretical work of Dietl et al. [1] magnetic semiconductors have been attracting remarkable attention in the spintronics community due to the possibility of the creation of new semiconductor spintronics devices. Even though not all expectations did materialize so far the interest in such materials is still rather high $[2,3]$. Recent theoretical works on magnetic semiconductors have been mostly focused on the origin of ferromagnetic ordering $[1,2,4-7]$, magnetic anisotropy [812 , and the effect of strain on the energy spectrum and magnetic anisotropy [13-15].

The most important of magnetic semiconductors is GaAs heavily doped with Mn ions, which is ferromagnetic at temperatures below $110 \mathrm{~K}$. It turns out that the doping with Mn makes this semiconductor of $p$-type for conductivity which substantially complicates the theoretical description of its electronic, magnetic and transport properties. It concerns the case of 3D bulk material and quasi-2D thin film on a substrate. In the latter case, any standard approach based on the model of 2D electron gas with the Rashba or Dresselhaus spin-orbit interaction cannot be used due to the strong inter-subband coupling of holes in the valence band. In other words, the electronic structure of $p$-type semiconductor should be presented by a set of strongly coupled energy bands.

\section{Model}

As a starting point we use the standard six-band Kane model of the valence band in 3D semiconductor [5], which is based on $k \cdot p$ approximation of electron energy spectrum [16-18]. The basis functions of this model are the eigenfunctions of the total momenta $J=3 / 2$ and $J=1 / 2$ at the $\Gamma$ point of the Brillouin zone. The Hamiltonian of holes in this model is the following $6 \times 6$ matrix [5]:

$$
\hat{H}_{0}=-E_{0}\left(\begin{array}{cccccc}
P+Q & L & M & 0 & \mathrm{i} L / \sqrt{2} & -\mathrm{i} \sqrt{2} M \\
L^{*} & P-Q & 0 & M & -\mathrm{i} \sqrt{2} Q & \mathrm{i} \sqrt{3 / 2} L \\
M^{*} & 0 & P-Q & -L & -\mathrm{i} \sqrt{3 / 2} L^{*} & -\mathrm{i} \sqrt{2} Q \\
0 & M^{*} & -L^{*} & P+Q & -\mathrm{i} \sqrt{2} M^{*} & -\mathrm{i} L^{*} / \sqrt{2} \\
-\mathrm{i} L^{*} / \sqrt{2} & \mathrm{i} \sqrt{2} Q & \mathrm{i} \sqrt{3 / 2} L & \mathrm{i} \sqrt{2} M & P+\Delta_{s o} / E_{0} & 0 \\
\mathrm{i} \sqrt{2} M^{*} & -\mathrm{i} \sqrt{3 / 2} L^{*} & \mathrm{i} \sqrt{2} Q & \mathrm{i} L / \sqrt{2} & 0 & P+\Delta_{s o} / E_{0}
\end{array}\right) \text {, }
$$

where $E_{0}=\hbar^{2} / 2 m_{0}$ and $\Delta_{s o}$ is split-off band gap and $m_{0}$ is the free electron mass,

$$
\begin{aligned}
& P=\gamma_{1}\left(k_{x}^{2}+k_{y}^{2}+k_{z}^{2}\right), \quad Q=\gamma_{2}\left(k_{x}^{2}+k_{y}^{2}-2 k_{z}^{2}\right), \\
& L=-2 \sqrt{3} \mathrm{i} \gamma_{3}\left(k_{x}-\mathrm{i} k_{y}\right) k_{z}, \quad M=\sqrt{3}\left[\gamma_{2}\left(k_{x}^{2}-k_{y}^{2}\right)-2 \mathrm{i} \gamma_{3} k_{x} k_{y}\right]
\end{aligned}
$$

and $\gamma_{1}=6.85, \gamma_{2}=2.1, \gamma_{3}=2.9$ are material specific Luttinger parameters for GaAs.

The magnetization related to the ferromagnetic ordering of magnetic moments of Mn ions, which are introduced to GaAs by doping, can be presented by the magnetic contribution to the Hamiltonian of holes [5]: 


$$
\hat{H}_{m}=B_{G}\left(\begin{array}{cccccc}
3 n_{z} & \mathrm{i} \sqrt{3} n_{-} & 0 & 0 & \sqrt{6} n_{-} & 0 \\
-\mathrm{i} \sqrt{3} n_{+} & n_{z} & 2 \mathrm{i} n_{-} & 0 & \mathrm{i} 2 \sqrt{2} n_{z} & -\sqrt{2} n_{-} \\
0 & -2 \mathrm{i} n_{+} & -n_{z} & \mathrm{i} \sqrt{3} n_{-} & \sqrt{2} n_{+} & -\mathrm{i} 2 \sqrt{2} n_{z} \\
0 & 0 & -\mathrm{i} \sqrt{3} n_{+} & -3 n_{z} & 0 & -\sqrt{6} n_{+} \\
\sqrt{6} n_{+} & -\mathrm{i} 2 \sqrt{2} n_{z} & \sqrt{2} n_{-} & 0 & -n_{z} & \mathrm{i} n_{-} \\
0 & -\sqrt{2} n_{+} & \mathrm{i} 2 \sqrt{2} n_{z} & -\sqrt{6} n_{-} & -\mathrm{i} n_{+} & n_{z}
\end{array}\right)
$$

where $B_{G}=\beta N_{0} M_{0} / 6 g \mu_{B}$ is the spin splitting parameter, $g=2$ and $\beta N_{0}=(-1.2 \pm 0.2) \mathrm{eV}$ for GaAs, $\mu_{B}=$ $e \hbar / 2 m_{0}$, where $\mu_{B}$ is the Bohr magneton. We denote the component of unit vector $\mathbf{n}$ determining the direction of magnetization $\mathbf{M}, n_{z}=M_{z} / M_{0}, n_{ \pm}=\left(M_{x} \pm \mathrm{i} M_{y}\right) / M_{0}$. The transition to $2 \mathrm{D}$ model can be done by introducing the quantization of wave-vector component $k_{z}=\pi / L$.

The Rashba spin-orbit interaction can be calculated by using the basis functions of the Kane model [16]:

$$
\hat{H}_{R}=\alpha_{s o}\left(\begin{array}{cccccc}
1 & 0 & 0 & 0 & 0 & 0 \\
0 & -\frac{1}{3} & 0 & 0 & \mathrm{i} \frac{\sqrt{2}}{3} & 0 \\
0 & 0 & -\frac{1}{3} & 0 & 0 & \mathrm{i} \frac{\sqrt{2}}{3} \\
0 & 0 & 0 & 1 & 0 & 0 \\
0 & -\mathrm{i} \frac{\sqrt{2}}{3} & 0 & 0 & -\frac{2}{3} & 0 \\
0 & 0 & -\mathrm{i} \frac{\sqrt{2}}{3} & 0 & 0 & -\frac{2}{3}
\end{array}\right)
$$

where $\alpha_{s o}$ is the Rashba coupling strength.

We also calculated the components of the spin operator using the basis of the Hamiltonian (1). For this purpose we used the explicit form of the basis functions from Ref. [16]. They are presented by the following matrices:

$$
\begin{aligned}
\hat{S}_{x} & =\left(\begin{array}{cccccc}
0 & \frac{\mathrm{i}}{\sqrt{3}} & 0 & 0 & \sqrt{\frac{2}{3}} & 0 \\
-\frac{\mathrm{i}}{\sqrt{3}} & 0 & \frac{2}{3} \mathrm{i} & 0 & 0 & -\frac{\sqrt{2}}{3} \\
0 & -\frac{2}{3} \mathrm{i} & 0 & \frac{\mathrm{i}}{\sqrt{3}} & \frac{\sqrt{2}}{3} & 0 \\
0 & 0 & -\frac{\mathrm{i}}{\sqrt{3}} & 0 & 0 & -\sqrt{\frac{2}{3}} \\
\sqrt{\frac{2}{3}} & 0 & \frac{\sqrt{2}}{3} & 0 & 0 & \frac{\mathrm{i}}{3} \\
0 & -\frac{\sqrt{2}}{3} & 0 & -\sqrt{\frac{2}{3}} & -\frac{\mathrm{i}}{3} & 0
\end{array}\right), \\
\hat{S}_{y} & =\left(\begin{array}{cccccc}
0 & \frac{1}{\sqrt{3}} & 0 & 0 & -\sqrt{\frac{2}{3}} \mathrm{i} & 0 \\
\frac{1}{\sqrt{3}} & 0 & \frac{2}{3} & 0 & 0 & \frac{\sqrt{2}}{3} \mathrm{i} \\
0 & \frac{2}{3} & 0 & \frac{1}{\sqrt{3}} & \frac{\sqrt{2}}{3} \mathrm{i} & 0 \\
0 & 0 & \frac{1}{\sqrt{3}} & 0 & 0 & -\sqrt{\frac{2}{3}} \mathrm{i} \\
\sqrt{\frac{2}{3}} \mathrm{i} & 0 & -\frac{\sqrt{2}}{3} \mathrm{i} & 0 & 0 & \frac{1}{3} \\
0 & -\frac{\sqrt{2}}{3} \mathrm{i} & 0 & \sqrt{\frac{2}{3}} \mathrm{i} & \frac{1}{3} & 0
\end{array}\right),
\end{aligned}
$$

$$
\hat{S}_{z}=\left(\begin{array}{cccccc}
1 & 0 & 0 & 0 & 0 & 0 \\
0 & \frac{1}{3} & 0 & 0 & \frac{2 \sqrt{2}}{3} \mathrm{i} & 0 \\
0 & 0 & -\frac{1}{3} & 0 & 0 & -\frac{2 \sqrt{2}}{3} \mathrm{i} \\
0 & 0 & 0 & -1 & 0 & 0 \\
0 & -\frac{2 \sqrt{2}}{3} \mathrm{i} & 0 & 0 & -\frac{1}{3} & 0 \\
0 & 0 & \frac{2 \sqrt{2}}{3} \mathrm{i} & 0 & 0 & \frac{1}{3}
\end{array}\right) .
$$

To find the expectation value of spin in a certain state of hole, we average the spin operators $\left\langle S_{\alpha}\right\rangle=\left\langle n \boldsymbol{k}\left|\hat{S}_{\alpha}\right| n \boldsymbol{k}\right\rangle$, where $|n \boldsymbol{k}\rangle$ is the eigenstate of the Hamiltonian describing the magnetized 2D system with Rashba spin-orbit coupling, $\hat{H}=\hat{H}_{0}+\hat{H}_{m}+\hat{H}_{R}$, with $k_{z}=\pi / L$.

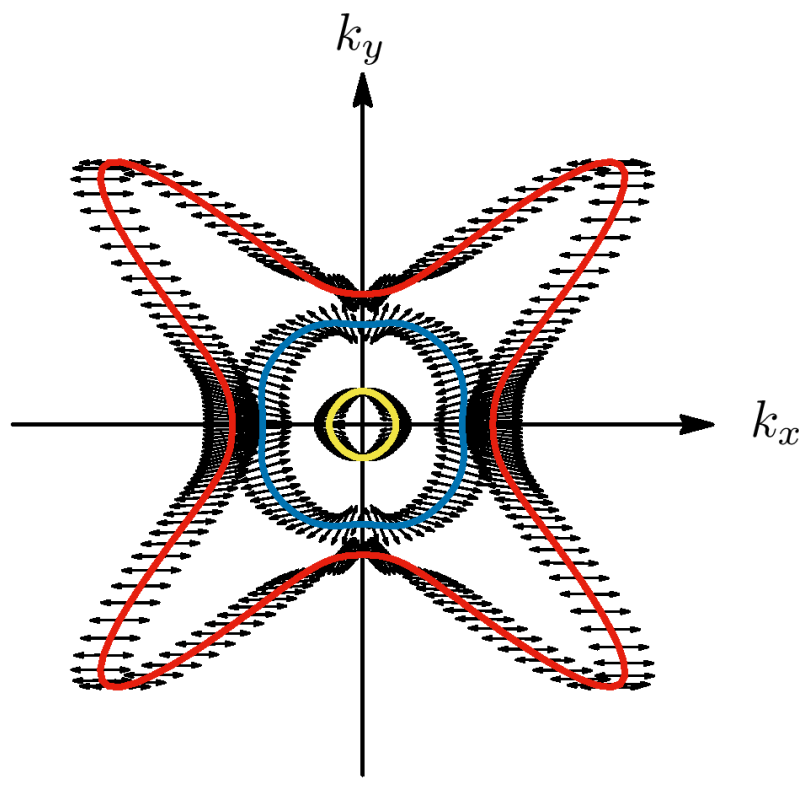

Fig. 1. Spin projection in $x y$-plane at $k_{z}=\pi / 10 \mathrm{~nm}$ with isoenergy $\mu=-0.5 \mathrm{eV}, B_{G}=0.1 \mathrm{meV}, \boldsymbol{M} \| \hat{x}$.

\section{Results}

The isoenergy contour for top valence bands, originating from the light holes (blue), heavy holes (red) and spin-split holes is shown in Fig. 1. Here we use very small magnitude of the magnetization parameter $B_{G}$, so that the magnetization-indices splitting of the light and heavy hole bands can be neglected in this schematic picture. The isoenergy contours in Fig. 1 are calculated for the case of zero Rashba coupling, $\alpha_{s o}=0$. In the figure we schematically indicated the orientation of spin vector calculated as an average value $\left\langle S_{\alpha}\right\rangle$. 


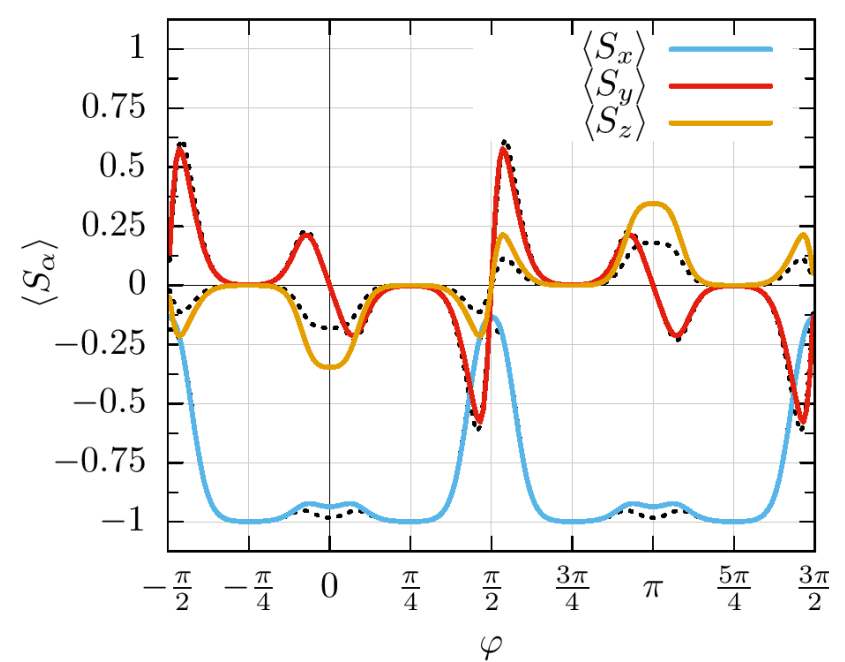

Fig. 2. Spin polarization components in the heavy-hole band as a function of the angle $\varphi$ in the $k_{x}-k_{y}$ plane (see Fig. 1). The dotted lines correspond to the spinpolarization without Rashba coupling, see Fig. 1. The solid lines are for the Rashba coupling $\alpha_{s o}=0.1 \mathrm{eV} \mathrm{m}$.

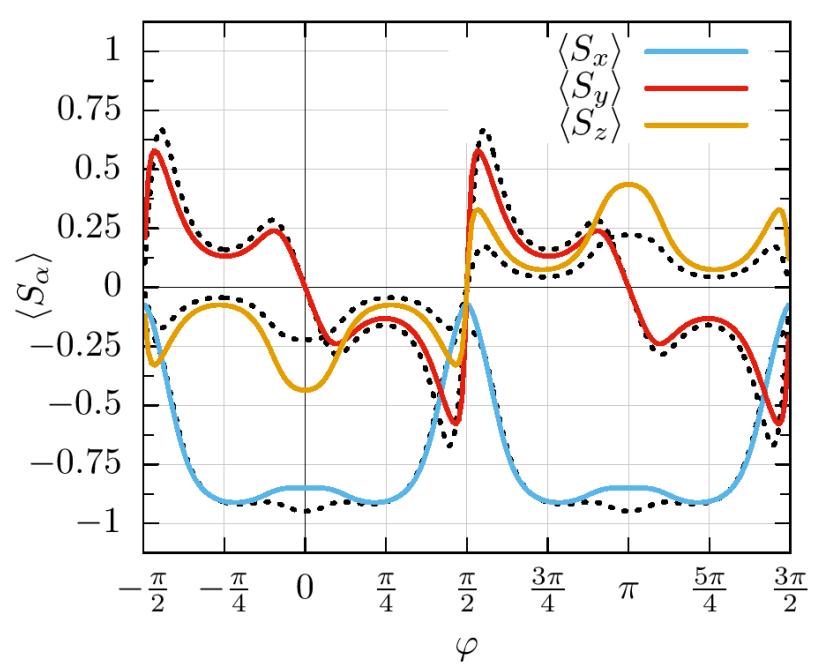

Fig. 3. Spin components for the light-hole band as a function of $x y$-plane angle $\varphi$. The dotted lines corresponds to $\alpha_{s o}=0$, the solid lines are for $\alpha_{s o}=0.1 \mathrm{eV} \mathrm{m}$.

The value of the spin polarization components of heavy holes at the same energy is shown in Fig. 2, for the light holes it is shown in Fig. 3, and for the split-off bands is shown in Fig. 4. At the small magnitude of $B_{G}$, all the eigenstates are nearly two-folded, where the second value in the pair has an opposite sign of $\left\langle S_{\alpha}\right\rangle$ and is not shown.

As we see, the Rashba spin-orbit coupling is mostly affecting the spin states in the spin-split band. In the light and hole band the substantial changes are for some orientations of the vector $\boldsymbol{k}$ with respect to the crystallographic axes.

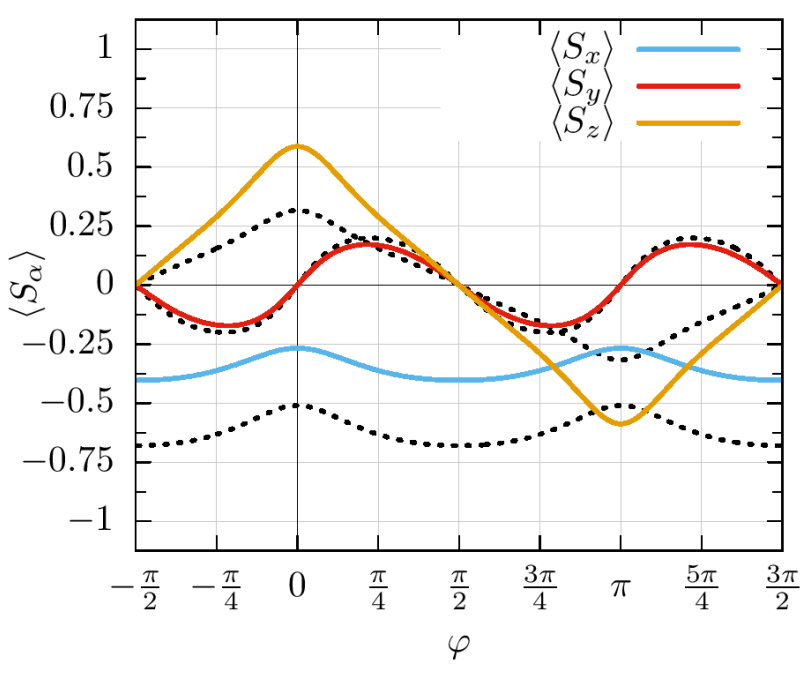

Fig. 4. Spin components for the spin-split band as a function of $x y$-plane angle $\varphi$. The dotted lines corresponds to $\alpha_{s o}=0$, the solid lines are for $\alpha_{s o}=0.1 \mathrm{eV} \mathrm{m}$.

\section{Conclusions}

We calculated the variation of the expectation value of the spin of holes in the valence band of two-dimensional GaMnAs magnetic semiconductor due to the Rashba spin-orbit interaction related to the substrate. We found that the Rashba spin-orbit field can substantially change the magnitude and orientation of the effective spin. This can be very important for spin-resolved transport phenomena in GaMnAs thin magnetic layers.

\section{Acknowledgments}

This work is supported by National Science Center in Poland as a research project No. DEC2012/06/M/ST3/00042.

\section{References}

[1] T. Dietl, H. Ohno, F. Matsukura, J. Cibert, D. Ferrand, Science 287, 1019 (2000).

[2] T. Jungwirth, Jairo Sinova, J. Mašek, J. Kucera, A.H. MacDonald, Rev. Mod. Phys. 78, 809 (2006).

[3] T. Dietl, H. Ohno, Rev. Mod. Phys. 86, 187 (2014).

[4] T. Dietl, A. Haury, Y. Merle d'Aubigne, Phys. Rev. $B$ 55, 6 (1997).

[5] T. Dietl, H. Ohno, F. Matsukura, Phys. Rev. B 63, 195205 (2001).

[6] V.I. Litvinov, V.K. Dugaev, Phys. Rev. Lett. 86, 5593 (2001).

[7] S.C. Erwin, A.G. Petukhov, Phys. Rev. Lett. 89, 227201 (2002). 
[8] M. Abolfath, T. Jungwirth, J. Brum, A.H. MacDonald, Phys. Rev. B 63, 054418 (2001).

[9] M. Sawicki, F. Matsukura, A. Idziaszek, T. Dietl, G.M. Schott, C. Ruester, C. Gould, G. Karczewski, G. Schmidt, L.W. Molenkamp, Phys. Rev. B 70, 245325 (2004).

[10] J. Zemen, J. Kucera, K. OlejnĂk, T. Jungwirth, Phys. Rev. B 80, 155203 (2009).

[11] W. Stefanowicz, C. Śliwa, P. Aleshkevych, T. Dietl, M. Döppe, U. Wurstbauer, W. Wegscheider, D. Weiss, M. Sawicki, Phys. Rev. B 81, 155203 (2010).

[12] S. Stagraczyński, C. Jasiukiewicz, V.K. Dugaev, J. Berakdar, J. Magn. Magn. Mater. 411, 79 (2016).

[13] J. Wunderlich, A.C. Irvine, J. Zemen, V. Holý, A.W. Rushforth, E. De Ranieri, U. Rana, K. Výborný, J. Sinova, C.T. Foxon, R.P. Campion, D.A. Williams, B.L. Gallagher, T. Jungwirth, Phys. Rev. B 76, 054424 (2007).
[14] L. Dreher, D. Donhauser, J. Daeubler, M. Glunk, C. Rapp, W. Schoch, R. Sauer, W. Limmer, Phys. Rev. B 81, 245202 (2010).

[15] C.S. King, J. Zemen, K. Olejník, L. Horák, J.A. Haigh, V. Novák, A. Irvine, J. Kucera, V. HolĂ", R.P. Campion, B.L. Gallagher, T. Jungwirth, Phys. Rev. B 83, 115312 (2011).

[16] G.L. Bir, G.E. Pikus, Symmetry and Strain-Induced Effects in Semiconductors, Wiley, New York 1974.

[17] R. Winkler, Spin-Orbit Coupling Effects in TwoDimensional Electron and Hole-Systems, Springer, Berlin 2003.

[18] L.C. Voon, M. Willatzen, The $\boldsymbol{k} \cdot \boldsymbol{p}$ Method. Electronic Properties of Semiconductors, Springer, Berlin 2009. 